\title{
Anatomy-Preserving Nonlinear Registration of Deep Brain ROIs Using Confidence-Based Block-Matching
}

\author{
Manik Bhattacharjee ${ }^{1,2}$, Alain Pitiot $^{3}$, Alexis Roche ${ }^{4}$, Didier Dormont ${ }^{1,2}$, \\ and Eric Bardinet ${ }^{1,2,5}$ \\ ${ }^{1}$ CNRS UPR640 - LENA, Paris, France \\ manik.bhattacharjee@chups.jussieu.fr \\ ${ }^{2}$ Université Paris 6, Paris, France \\ ${ }^{3}$ LIDA, Brain \& Body Centre, University of Nottingham, UK \\ ${ }^{4}$ CEA Neurospin, Gif-sur-Yvette, France \\ ${ }^{5}$ CENIR, Paris, France
}

\begin{abstract}
Brain atlases are commonly used in a number of applications such as MRI segmentation and surgery targetting. Our goal is to register a basal ganglia atlas to a subject using MR image registration. Existing registration methods are for the most part either too constrained (linear registration) or can deform deep brain ROIs into implausible anatomical shapes.

We developed a block-matching registration method suitable for atlas registration, using a new confidence-based regularization of the vector field. The method was used to register a set of 17 manually segmented MRI onto one subject. Results show that basal ganglia structures were better registered than when using an affine registration method.
\end{abstract}

\section{Introduction}

Brain atlases are very useful tools in neuroimaging, and are commonly used in a number of applications, such as segmentation of brain structures in a MRI scan, neurosurgery targetting, or to compare functional activity of a group of subjects on a common anatomy.

Our goal is to register a 3D histological atlas of the basal ganglia [1, composed of a T1-weighted MRI and a set of finely defined regions of interest (ROIs) to any subject T1-weighted MRI as precisely as possible while retaining an anatomically-plausible shape for each deep brain structure.

Numerous approaches 2] have been proposed to solve this intersubject brain registration problem. The main difficulty arises from the high variability of brain anatomy in intersubject brain registration, which is very visible in the cortex gyri and sulci, but also in the size and shape of the lateral ventricles.

The registration problem can be defined as finding the transformation $T$ in a transformation space $T S$ that transforms a floating image $I_{F l o a t}$ into the image space of a reference image $I_{R e f}$ while maximizing a similarity measure $S$ between $I_{\text {Ref }}$ and $T\left(I_{\text {float }}\right)$. 


$$
T=\underset{T \in T S}{\operatorname{argmax}} S\left(T\left(I_{\text {float }}\right), I_{\text {Ref }}\right)
$$

To constrain the deformation of brain structures, the simplest approach is to choose a limited transformation space $T S$, such as the space of affine transformations. This class of transformations cannot radically change the shape of deformed objects and is therefore suitable to the task. However, affine transformations are not sufficient to model local variations such as a different volume of the ventricles between two subjects with similar brain shapes. It is therefore necessary to increase the flexibility of the deformation.

Nonlinear algorithms have been developed to address this point. They can be roughly divided in two main classes: geometrical and iconic algorithms. Geometrical algorithm use data extracted from images to perform the registration, whereas iconic algorithms use only the intensity values of image voxels.

In geometrical algorithms, a segmentation step is needed before the registration. This segmentation has a major influence on the registration results and can be difficult. For example [3] uses segmented cortical sulci to register the brain image.

Iconic methods use image intensities to drive the registration. Elastic blockmatching algorithms such as 4] provide satisfactory results. Fluid methods allow to warp an image onto another but the resulting deformation field may contain high variations that when applied to ROI can lead to implausible anatomical shapes.

Because our main focus is to deform histological ROIs through MRI intersubject registration, we developed a new block-matching algorithm that offers a good trade-off between image matching quality and deformation field regularity. The novelty arises from the introduction of a matching confidence into the field regularization process.

The method was evaluated using a manually segmented set of T1-weighted MR images (IBSR database).

\section{Method}

\subsection{Block-Matching}

The algorithm registers an image $I_{\text {Float }}$ onto a target image $I_{\text {Ref }}$. $I_{\text {Float }}$ is divided into cubic blocks of voxels.

Each block $b_{i, j, k}$ is compared to blocks of the same dimensions in the reference image in the neighborhood of its position $(i, j, k)$. The restriction to a neighborhood $N_{i, j, k}$ in $I_{R e f}$ constrains the solution to a realistic subset of positions and limits the risk of finding erroneous matches in distant parts of the brain, as the images are typically already affine-registered before using our nonlinear method.

To compare $b_{i, j, k}$ to a block in $I_{R e f}$, a number of similarity measures is available in the literature, such as the sum of squared differences (SSD), the correlation coefficient, the mutual information and its variants. SSD is sensitive to bias in the images and intensity differences between images, and so is not best 

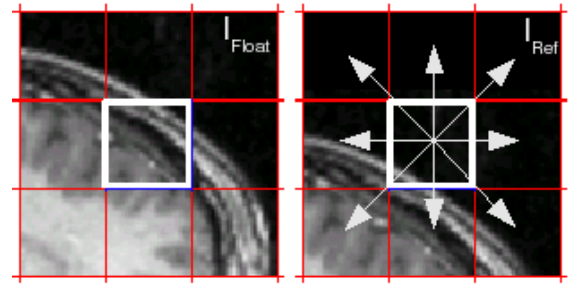

$\begin{array}{lll}0.01 & 0.01 & 0.01\end{array}$

$\begin{array}{lll}0.07 & 0.05 & 0.01\end{array}$

$\begin{array}{lll}0.1 & 0.6 & 0.02\end{array}$

Similarity Map of the block

Fig. 1. Block-matching and BSM construction

\begin{tabular}{|lll||l|lll|}
\hline 0.01 & 0.01 & 0.01 \\
0.07 & 0.05 & 0.01 \\
0.1 & 0.6 & 0.02 & & 0.01 & 0.959 & 0.01 \\
0.2 & 0.959 & 0.01 \\
0.3 & 0.96 & 0.02
\end{tabular}

Fig. 2. Two BSM with the same best vector, with moderate scalar and high matrix confidence (left), high scalar and low matrix confidence (right). The uncertainty ellipsoid (inverse of the matrix confidence) is displayed along the vector.

suited for a registration involving images from various scanners. Mutual information can model nonlinear relationships between the voxels of two images. This measure is less robust than correlation as shown in [5] and its properties are not needed in an intra-modality registration, where the correlation coefficient, defined as the covariance of the two blocks divided by their respective variance, is sufficient:

$$
c c(b 1, b 2)=\frac{\operatorname{cov}(b 1, b 2)}{\sigma_{b 1} \cdot \sigma_{b 2}}
$$

Each block $b_{i, j, k}$ is compared to a number of potential matching positions in $I_{\text {Ref }}$. A 3D block similarity map (BSM) is created, composed of the similarity values for all the possible positions of the block $b_{i, j, k}$ in a neighborhood $N_{i, j, k}$ in $I_{\text {Ref }}$ (see figure 1).

In the BSM, the displacement corresponding to the maximum similarity value is chosen as the displacement vector $\boldsymbol{v}$ for the block.

$$
\boldsymbol{v}=\underset{(u, v, w) \in N_{i, j, k}}{\operatorname{argmax}} B S M(u, v, w)
$$

This way, a vector field with one vector $\boldsymbol{v}=\left(u_{c c}, v_{c c}, w_{c c}\right)$ for each image block is defined.

\subsection{Confidence}

The raw deformation field obtained during block-matching cannot be used directly as it is usually rather noisy and chaotic. Therefore, most existing methods smooth the raw field, e.g. using a linear filter such as the Gaussian filter, or a 
median filter [4], or via fitting a parametric deformation model such as a local linear model 6].

All these smoothing methods are data-unrelated: all vectors are considered equally relevant in the search for the best transformation. In contrast, the present method regularizes the displacement field by weighting each vector according to a measure of confidence. The notion of confidence can be understood in two distinct ways:

Matching quality: a scalar value assessing the quality of the match between the block and its target block in the reference image. This scalar confidence is a probability-like value that stems directly from the similarity between the two blocks as measured by the correlation coefficient. The correlation coefficient $c c$ was shown in [7] to relate with the profile likelihood of the transformation parameters via:

$$
L \propto\left(1-c c^{2}\right)^{-\frac{n}{2}}
$$

where $n$ is the number of voxels used to compute $c c$. This formula gives the likelihood of a match in the limit of large $n$. In practice, numerical instabilities may occur for small blocks as $c c$ tends to be overestimated. To avoid these, we use a first-order Taylor expansion of the log-likelihood, yielding: $L \propto e^{\frac{n}{2} c c^{2}}$.

Confidence in the chosen displacement: Interpreting $L$ as a probability distribution on block displacements (up to normalization constant), we see that the most probable displacement is the one maximizing $c c$. However, Figure 2 shows that the same vector can be chosen with very different BSM. In the first case, the chosen vector is probably the best solution, but in the second BSM, the difference between the two maxima shows that the vector does not provide reliable information on the real transformation.

As proposed in [8] with another confidence measure, the BSM distribution of block $b_{i, j, k}$ around the chosen vector can be characterized by the covariance matrix of the probability BSM around the chosen vector $\left(u_{c c}, v_{c c}, w_{c c}\right)$ :

$$
\operatorname{Cov}(i, j, k)=\left(\begin{array}{ccc}
S_{u, u} & S_{u, v} & S_{u, w} \\
S_{u, v} & S_{v, v} & S_{v, w} \\
S_{u, w} & S_{v, w} & S_{w, w}
\end{array}\right)
$$

with:

$$
S_{u, v}=\frac{\sum_{(u, v, w) \in N_{i, j, k}} L(u, v, w)\left(u-u_{c c}\right)\left(v-v_{c c}\right)}{\sum_{(u, v, w) \in N_{i, j, k}} L(u, v, w)},
$$

where $L(u, v, w)$ is the BSM likelihood value at $(u, v, w)$. This can be understood as a $3 \mathrm{D}$ ellipsoid of uncertainty around the vector (see figure 2).

Both types of confidence are relevant and complementary (figure 2 shows two unreliable vectors due to either a low scalar confidence or to a low matrix confidence). They are used in the regularization process.

\subsection{Regularization of the Vector Field}

Regularization of the vector field is performed on a vector-by-vector basis. For each vector $\boldsymbol{v}$, a cubic neighborhood of vectors $V N(v)$ is defined, and all the 
vectors in the neighborhood contribute to compute a new value for the regularized vector $\hat{\boldsymbol{v}}$. This step will increase the local smoothness of the vector field, and is therefore essential to the anatomically-correct deformation of subcortical structures.

Matrix Confidence. As proposed by Singh and Allen 8 , the matrix confidence associated to each vector can be used to provide a mean vector weighted by the 3D confidence. The mean vector is the Mahalanobis-distance-weighted sum of all the vectors in the neighborhood.

$$
\hat{\boldsymbol{v}}=\left(\sum_{\boldsymbol{u} \in V N(v)} \operatorname{Cov}^{-1}(\boldsymbol{u}) \cdot(u)\right) \cdot\left(\sum_{\boldsymbol{u} \in V N(v)} \operatorname{Cov}^{-1}(\boldsymbol{u})\right)^{-1}
$$

The matrices that must be inverted are real and symmetric, and are therefore inverted using their eigenvalues decomposition.

Scalar Confidence. The scalar confidence $c=L\left(u_{c} c, v_{c} c, w_{c} c\right)$ can be used in a similar way as a weight for each vector.

$$
\hat{\boldsymbol{v}}=\frac{\sum_{\boldsymbol{u} \in V N(v)} c(\boldsymbol{u}) \cdot \boldsymbol{u}}{\sum_{\boldsymbol{u} \in V N(v)} c(\boldsymbol{u})}
$$

BSM Filtering. In order to better control the influence of both confidences on the regularization, we chose to filter the Block Similarity Maps before extracting confidence values to mitigate the effect of the exponential from the likelihood formula which transforms a slight difference of similarity between two good matches into a large difference. BSM were thus filtered $\widehat{B S M}(i, j, k)=B S M^{f}(i, j, k)$ where $\mathrm{f}$ is a parameter of the filter with a value between 0 and 1 .

Gaussian Weight. Using only confidence-based regularization will not provide a smooth regularization field: if there is one high-confidence vector $\boldsymbol{V}=(1,0,0)$ in a vector field composed of low-confidence vectors $(0,0,1)$, there will be a discontinuity in the regularized vector field around $\boldsymbol{V}$, as all vectors with $\boldsymbol{V}$ in their neighborhood will be almost equal to $(1,0,0)$, whereas vectors without $\boldsymbol{V}$ in their neighborhood will be equal to $(0,0,1)$. A way to solve this is to give a small weight to distant vectors of the neighborhood. We chose a Gaussian centered on the vector $v$ using the euclidean distance $d$ between the vector and its neighbor as a parameter:

$$
\hat{\boldsymbol{v}}=\frac{\sum_{\boldsymbol{u} \in V N(v)} g(d(u, v)) \cdot \boldsymbol{u}}{\sum_{\boldsymbol{u} \in V N(v)} g(d(u, v))}
$$

Full Regularization. Gaussian $g$, scalar confidence $c$ and matrix confidence $\operatorname{Cov}^{-1}(\boldsymbol{u})$ regularization are combined in our method:

$\hat{\boldsymbol{v}}=\left(\sum_{\boldsymbol{u} \in V N(v)} \operatorname{Cov}^{-1}(\boldsymbol{u}) \cdot g(d(u, v)) \cdot c(u) \cdot \boldsymbol{u}\right) \cdot\left(\sum_{\boldsymbol{u} \in V N(v)} g(d(u, v)) \cdot c(u) \cdot \operatorname{Cov}^{-1}(\boldsymbol{u})\right)^{-1}$ 


\subsection{Pyramidal and Iterative Registration}

For robustness, the algorithm is applied in a multiscale, coarse-to-fine image resolution pyramid: the images are registered at an increasing resolution at each step of the pyramid. This allows a better convergence of the registration towards the global optimum, and a diminished risk of it getting trapped in a local optimum.

To increase the accuracy of the algorithm, it is also repeated through a number of iterations at each step of the pyramid.

\subsection{Evaluation of the Registration}

To evaluate our registration method, we used the IBSR database 11 of 18 manually segmented T1-weighted brain MRIs of subjects ranging from juvenile to 71 years old.

We registered 17 of the 18 brains onto the last one. The images were first registered using baladin software 9 in three steps: rigid, similitude, and affine registrations. All images were resampled to the resolution and size of the target image with the final affine transformation.

The parameters chosen for the nonlinear registration were chosen according to image resolution. Blocks were adjacent and their size was $5 \times 5 \times 5$ voxels. The neighborhood explored for each block was 9x9x9 block positions in the reference image each spaced by a voxel. Four levels of the resolution pyramid were used: $1 / 8,1 / 4,1 / 2$ and $1 / 1$. At each level, 10 iterations were performed, enough to get a stable transformation at each level.
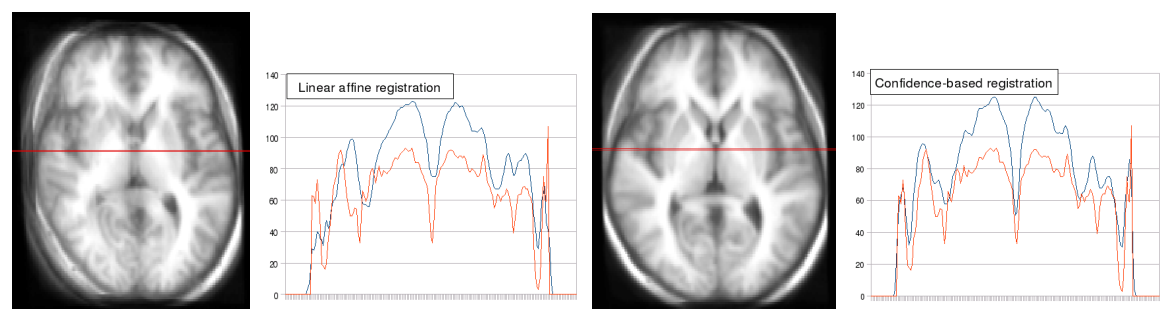

Fig. 3. Mean of the 17 resampled image using affine registration (left) and confidencebased registration (right), and intensity profile of the red line compared to the target image profile

Parameters screening on two high-resolution MR images was used to determine best BSM filter, Gaussian sigma and regularization neighborhood radius. The best results were obtained using a BSM filter parameter $f=0.24$, a regularization neighborhood of $7 \times 7 \times 7$ vectors and a Gaussian sigma such as the most distant vector was at $3 \sigma$ from the Gaussian center.

${ }^{1}$ The MR brain data sets and their manual segmentations were provided by the Center for Morphometric Analysis at Massachusetts General Hospital and are available at http://www.cma.mgh.harvard.edu/ibsr/ 


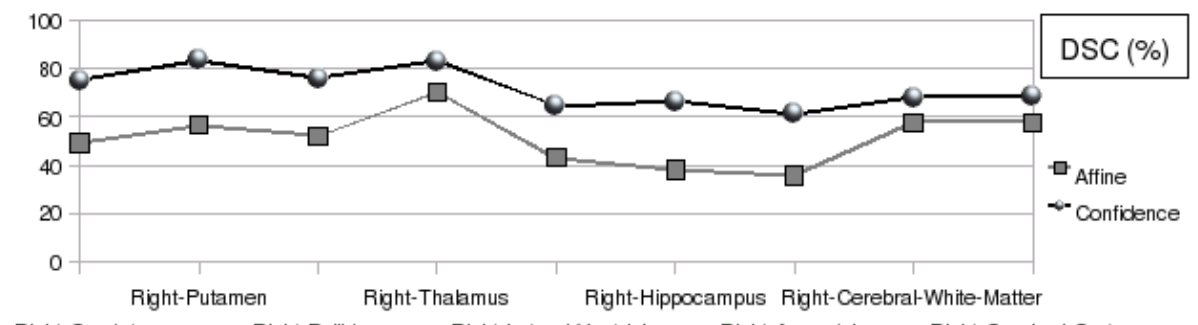

Right-Caudate Right-Pallidum Right-Laterat-Ventricle Right-Amygdaa Right-Cerebrat-Cortex

Fig. 4. Mean on all subjects of the DSC overlap index between registered segmentation and manual segmentation of the target brain

Mean of the 17 images was computed after affine registration and after confidence-based nonlinear registration.

The manual segmentation images of all 17 subjects were resampled using both registration methods and were compared to the manual segmentation of the target image using the Dice Similarity Coefficient (DSC) as used in [10:

$$
D S C=\frac{2 N(A \cap B)}{N(A)+N(B)}
$$

where $N(A)$ is the number of voxels in label A.

\section{Results}

Nonlinear registration was fast: 8 minutes were necessary to register each image on a bi-Intel Xeon processor equipped with $4 \mathrm{~GB}$ of memory.

The mean of the 17 resampled images using linear and confidence-based methods is displayed on figure 3. The confidence-based registration mean image is much sharper than the affine registration mean image. On the intensity profile (the intensity values corresponding to the red line on the average image) peaks are sharper for the skull, the ventricles and the white matter between ventricles with the confidence-based method. It is even possible to see the intensity values for some gray nuclei.

The mean across all subjects of DSC overlap index for right-side deep brain structures is plotted in figure 4 after linear and confidence-based registration. Confidence-based registration improves linear registration DSC overlap index by $44 \%$.

Using confidence indices allows the regularization process to keep more of the block-matching information in the final field. The confidence-based registration method gives promising results on a real set of data, and the transformation it provides is smooth enough to preserve anatomical shapes of the registered ROIs.

In future work, this method will be compared to other non-linear registration methods such as median regularized block-matching [4] or HAMMER [1]. 


\section{References}

1. Yelnik, J., Bardinet, E., Dormont, D., Malandain, G., Ourselin, S., Tandé, D., Karachi, C., Ayache, N., Cornu, P., Agid, Y.: A three-dimensional, histological and deformable atlas of the human basal ganglia. i. atlas construction based on immunohistochemical and MRI data. Neuro image 34, 618-638 (2007)

2. Zitova, B., Flusser, J.: Image registration methods: a survey. Image and Vision Computing 21(11), 977-1000 (2003)

3. Durrleman, S., Pennec, X., Trouvé, A., Ayache, N.: Measuring brain variability via sulcal lines registration: A diffeomorphic approach, pp. 675-682 (2007)

4. Lau, Y.H., Braun, M., Hutton, B.F.: Non-rigid image registration using a medianfiltered coarse-to-fine displacement field and a symmetric correlation ratio. Phys. Med. Biol. 46(4), 1297-1319 (2001)

5. Andronache, A., von Siebenthal, M., Székely, G., Cattin, P.: Non-rigid registration of multi-modal images using both mutual information and cross-correlation. Med. Image Anal. (2007)

6. Pitiot, A., Guimond, A.: Geometrical regularization of displacement fields for histological image registration. Med. Image Anal. (2007)

7. Roche, A., Malandain, G., Ayache, N.: Unifying Maximum Likelihood Approaches in Medical Image Registration. International Journal of Imaging Systems and Technology: Special issue on 3D imaging 11, 71-80 (2000)

8. Singh, A., Allen, P.: Image-flow computation: An estimation-theoretic framework and a unified perspective. IU 56, 152-177 (1992)

9. Ourselin, S., Roche, A., Subsol, G., Pennec, X., Ayache, N.: Reconstructing a 3d structure from serial histological sections. Image and Vision Computing 19(1-2), 25-31 (2001)

10. Crum, W.R., Camara, O., Hill, D.L.G.: Generalized overlap measures for evaluation and validation in medical image analysis. IEEE Trans. Med. Imaging 25(11), 1451$1461(2006)$

11. Shen, D., Davatzikos, C.: Hammer: hierarchical attribute matching mechanism for elastic registration. IEEE Trans. Med. Imaging 21(11), 1421-1439 (2002) 